\title{
A tale of three GTPases and a RIN in endothelial cell adhesion
}

\author{
Mar Fernandez-Borja ${ }^{1}$ \\ ${ }^{1}$ Department of Molecular Cell Biology, Sanquin Research and Landsteiner Laboratory, Academic Medical Center, University of \\ Amsterdam, Amsterdam, The Netherlands \\ Cell Research (2012) 22:1426-1428. doi:10.1038/cr.2012.118; published online 14 August 2012
}

Endothelial cell adhesion to the extracellular matrix regulates migration and outgrowth of blood vessels during angiogenesis. Cell adhesion is mediated by integrins, which transduce signals from the extracellular environment into the cell and, in turn, are regulated by intracellular signaling molecules. In a paper recently published in Cell Research, Sandri et al. show that RIN2 connects three GTPases, R-Ras, Rab5 and Rac1, to promote endothelial cell adhesion through the regulation of integrin internalization and Rac1 activation.

The formation of the vascular tree during development requires the orderly growth of blood vessels to irrigate all organs and tissues. This process of blood vessel remodeling, termed angiogenesis, requires endothelial cell proliferation, adhesion, migration and tube formation [1]. Pathological angiogenesis takes place during tumor growth as hypoxia within the tumor induces the release of pro-angiogenic mediators such as vascular endothelial growth factor (VEGF).

Small GTPases are critical for the regulation of cell behavior and thus also play a central role in angiogenesis. Small GTPases are 20-25 kDa signaling

Correspondence: Mar Fernandez-Borja

E-mail: m.fernandez@sanquin.nl proteins that cycle between an active GTP-bound and an inactive GDP-bound state. When active, GTPases associate with and activate diverse effector molecules that subsequently relay the signal to other molecules, ultimately leading to a specific cell response. Two classes of proteins facilitate GTPase cycling. Guanine exchange factors (GEFs) catalyze GDP unloading thereby promoting GTP binding and GTPase activation. Conversely, GTPase activating proteins (GAPs) enhance the intrinsic GTP hydrolysis activity of the GTPase leading to its inactivation. Small GTPases form a large superfamily with over 100 members in mammals. Based on structural and functional criteria, the GTPase superfamily is subdivided in Ras, Rab, Rho, Arf and Ran subfamilies, each of them generally, but not exclusively, specialized in the regulation of specific cellular events. For example, Rho GTPases primarily regulate cytoskeletal dynamics; Rab GTPases regulate intracellular membrane trafficking; and Ras GTPases function in the regulation of cell proliferation and survival. However, complex processes such as angiogenesis require the coordinated action of several GTPases. This is evidenced by the work of Sandri et al. [2] recently published in Cell Research. In their paper, Sandri et al. propose a mechanism for the regulation of endothelial cell adhesion and migration involving three
GTPases belonging to different GTPase branches, R-Ras, Rab5 and Rac1. The protein RIN2 (Ras and Rab adaptor 2) brings together R-Ras and Rab5 to form a signaling module that orchestrates integrin trafficking and Rac1 activation, processes that are essential for cell adhesion and migration.

Integrins are heterodimeric transmembrane extracellular matrix (ECM) receptors composed of one $\alpha$ and one $\beta$ chain. In a process known as 'outsidein' signaling, integrins transmit signals from the extracellular environment to intracellular adaptor and signaling molecules that regulate cell migration, survival and growth. Conversely, during 'inside-out' signaling, integrins can be switched from an inactive to an active conformation by cytoplasmic signaling molecules leading to increased integrin affinity for the ECM.

During 2D migration of adherent cells, nascent, highly dynamic focal contacts are formed at the leading edge lamellipodia where integrins mediate adhesion to the ECM. Some of these focal contacts disassemble and some mature into larger focal adhesions with a longer half-life. Failure in maintaining a dynamic assembly and disassembly of focal contacts will result in the inhibition of cell migration.

Integrin-mediated adhesion can be regulated at different levels: (1) by changing integrin conformation and 
thus affinity for their ligand; (2) by modulating integrin avidity, i.e., by promoting integrin clustering on the plasma membrane; and (3) by changing the kinetics of integrin endocytosis and/ or recycling [3].

The Ras GTPase R-Ras is primarily expressed in the vascular system (endothelial cells and vascular smooth muscle cells) [4]. Zhang et al. [5] were the first to show that R-Ras is a potent regulator of cell adhesion when they reported that expression of active R-Ras was enough to induce ECM adhesion of suspension cells, whereas dominant negative R-Ras reduced adhesion of the adherent cell line CHO. Although R-Ras was shown to enhance integrin affinity [5], this effect was not consistently observed $[6,7]$. These contradictory findings could be explained by the fact that R-Ras may activate integrins indirectly through antagonizing H-Ras-mediated integrin inhibition [6].

Recent findings suggest that R-Ras stimulates adhesion through the regulation of integrin internalization into Rab11-positive endosomes [8]. Now, the data of Sandri et al. [2] support this model. The authors addressed the question on how R-Ras regulates cell adhesion of endothelial cells by performing a yeast-two-hybrid screen using constitutively-active R-Ras as bait. The screen revealed that RIN2 is a major $\mathrm{R}-$ Ras-interacting protein. RIN proteins (RIN1, 2 and 3) are downstream effectors of Ras GTPases that function as GEFs for Rab5 [9], a GTPase that regulates endocytosis. RIN1 was shown to mediate the stimulation of EGF receptor-mediated endocytosis by $\mathrm{H}-$ Ras through the activation of Rab5 [10]. Surprisingly, Sandri et al. found that R-Ras dramatically impaired the Rab5 exchange activity of RIN2, while H-Ras had no effect. However, RIN2 was still able to specifically bind active Rab5. These data suggest that active R-Ras, RIN2 and active Rab5 form a signaling complex. Accordingly, Sandri et al. show that endogenous R-Ras, RIN2 and
Rab5 are indeed found in a complex in endothelial cells. While active R-Ras and RIN2 colocalize at nascent focal contacts and on intracellular vesicles, colocalization with Rab5 takes place on endosomes. The deletion of either the Ras- or the Rab5-binding domains of RIN2 prevented the colocalization of the trio. Thus, RIN2 appears to facilitate the transport of active R-Ras to Rab5-positive endosomes. What is the functional relevance of these interactions? Sandri et al. show that silencing of endogenous RIN2 impaired the increase in adhesion induced by active R-Ras and by Rab5. A similar effect was obtained upon expression of RIN2 deletion mutants lacking Ras- or Rab5-binding domains. These data strongly suggest that the adaptor function of RIN2 in connecting R-Ras and Rab5 regulates endothelial cell adhesion to the ECM. But what is the mechanism? Previous work has shown that the pro-adhesive activity of active $\mathrm{R}-\mathrm{Ras}$ is linked to its ability to regulate $\beta 1$ integrin endocytosis [8]. Sandri et $a l$. confirm these data by showing that silencing of R-Ras or RIN2 decreases the rate of endocytosis of active ECMengaged $\beta 1$ integrins. In addition, the authors set a step further as they show that the signaling complex R-Ras/RIN2/ Rab5 mediates basal Rac1 GTPase activation. Rac1 regulates actin dynamics and ruffle formation at the leading edge of migrating cells and its activity is essential for cell adhesion and migration. TIAM-1-mediated activation of Rac1 on endosomes and subsequent polarized transport to the plasma membrane has been proposed as a way to restrict Rac activity to sites of membrane protrusion $[11,12]$. In line with this model, Sandri et al. show that active R-Ras and RIN2 colocalize with Rac1 on endosomes and that the endosomal Rac GEF TIAM-1 is necessary for R-Ras- and RIN2-induced cell adhesion.

Altogether, the data of Sandri et al. support a model in which, integrinactivated R-Ras recruits RIN2 to focal adhesions and induces RIN2 conversion from a Rab5 GEF to a Rab5-docking protein. Subsequently, the complex promotes the endocytosis of ECM-engaged integrins and moves to early endosomes where R-Ras activates the TIAM-1/ Rac1 pathway [13]. Active Rac1 translocates to the plasma membrane where it promotes actin polymerization and formation of new focal contacts (Figure 1).

By bridging active R-Ras and Rab5, RIN2 combines two processes essential for cell adhesion: (1) focal contact dynamics through the internalization of ECM-engaged integrins; and (2) local Rac1 activation to ensure actin polymerization at lamellipodia. Similarly, RIN2 also connects H-Ras and Rab5 in the internalization of the epithelial cellcell adhesion molecule E-cadherin [14]. Thus, RIN2 appears to be a universal effector of Ras-induced endocytosis of membrane receptors.

Interestingly, the phenotype of a family with a homozygous mutation in RIN2 was recently described [15]. The affected individuals showed diverse abnormalities related to a defective connective tissue. Indeed, ultrastructural analysis of the skin showed an abnormal morphology of collagen fibrils. Collagen is a ligand for $\beta 1$ integrins. Through simultaneous binding to collagen and to the intracellular cytoskeleton, integrins contribute to the assembly of the ECM by transmitting contraction forces from the cell to the ECM. It is tempting to speculate that the phenotype of the patients lacking RIN2 is due to a deficient $\beta 1$ integrin function as found by Sandri et al. in their in vitro analysis. In addition, these patients bruise easily and present prolonged bleeding, which could be caused by deficient wound healing of blood vessels as a consequence of impaired R-Ras signaling.

It should be noted, however, that R-Ras knockout mice have no major defects in vascular development but respond with increased angiogenesis to stress conditions such as tumor implantation [4]. On the contrary, the in 


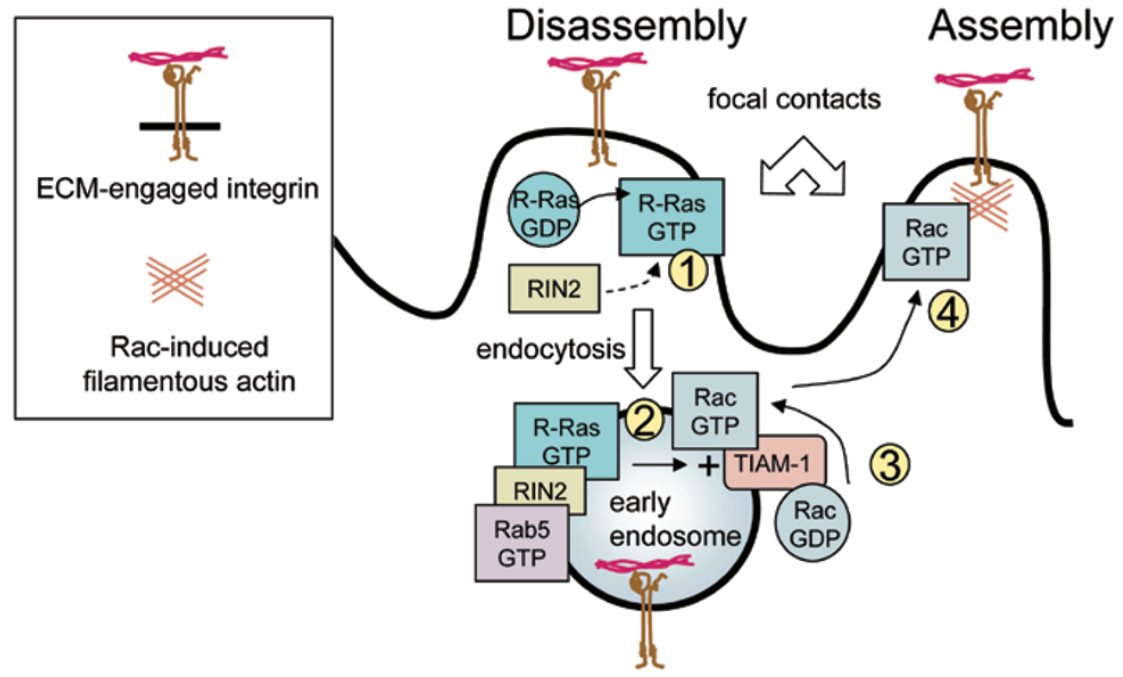

Figure 1 Model proposed by Sandri et al. [2] for the regulation of focal adhesion dynamics by R-Ras. (1) R-Ras is activated by ECM-engaged integrins, recruits RIN2 and converts it from a Rab5 GEF to a Rab5 adaptor; (2) RIN2 binding to active Rab5 mediates the endocytosis of integrins and the transport of active R-Ras to endosomes; (3) R-Ras contributes to the activation of the Rac1 GEF TIAM-1, which then activates Rac1; (4) Active Rac1 translocates to the plasma membrane and promotes actin polymerization and formation of new focal contacts.

vitro study by Sandri et al. suggests that R-Ras deficiency results in decreased endothelial cell migration. Further research is needed to clarify the role of RRas in angiogenesis. Likewise, it will be interesting to study vascular responses in RIN2-deficient mice in comparison to R-Ras knockout mice.

\section{Acknowledgments}

I would like to thank Dr Peter L Hordijk for critically reading the manuscript.

\section{References}

1 Heinke J, Patterson C, Moser M. Life is a pattern: vascular assembly within the embryo. Front Biosci 2012; 4:22692288.
2 Sandri C, Caccavari F, Valdembri D, et al. The R-Ras/RIN2/Rab5 complex controls endothelail cell adhesion and morphogenesis via active integrin endocytosis and Rac signaling. Cell Res 2012; 22:1479-1501.

3 Caswell PT, Vadrevu S, Norman JC. Integrins: masters and slaves of endocytic transport. Nat Rev Mol Cell Biol 2009; 10:843-853.

4 Komatsu M, Ruoslahti E. R-Ras is a global regulator of vascular regeneration that suppresses intimal hyperplasia and tumor angiogenesis. Nat Med 2005; 11:1346-1350.

5 Zhang Z, Vuori K, Wang H, Reed JC, Ruoslahti E. Integrin activation by Rras. Cell 1996; 85:61-69.

6 Sethi T, Ginsberg MH, Downward J, Hughes PE. The small GTP-binding protein R-Ras can influence integrin activation by antagonizing a Ras/Rafinitiated integrin suppression pathway. Mol Biol Cell 1999; 10:1799-1809.

7 Holly SP, Larson MK, Parise LV. The unique $\mathrm{N}$-terminus of $\mathrm{R}$-Ras is required for Rac activation and precise regulation of cell migration. Mol Biol Cell 2005; 16:2458-2469.

8 Conklin MW, Ada-Nguema A, Parsons M, Riching KM, Keely PJ. R-Ras regulates $\beta 1$-integrin trafficking via effects on membrane ruffling and endocytosis. BMC Cell Biol 2010; 11:14-28.

9 Bliss JM, Venkatesh V, Colicelly J. The RIN family of Ras effectors. Methods Enzymol 2006; 407:335-344.

10 Tall GG, Barbieri MA, Stahl PD, Horazdovsky BF. Ras-activated endocytosis is mediated by the Rab5 guanine nucleotide exchange activity of RIN1. Dev Cell 2001; 1:73-82.

11 Palamidessi A, Frittoli E, Garré M, et al. Endocytic trafficking of Rac is required for the spatial restriction of signaling in cell migration. Cell 2008; 134:135-147.

12 Osmani N, Peglion F, Chavrier P, Etienne-Manneville S. Cdc42 localization and cell polarity depend on membrane traffic. J Cell Biol 2010; 191:12611269.

13 Lambert JM, Lambert QT, Reuther GW, et al. Tiam1 mediates Ras activation of Rac by a PI(3)K-independent mechanism. Nat Cell Biol 2002; 4:621625.

14 Kimura T, Sakisaka T, Baba T, Yamada $\mathrm{T}$, Takai Y. Involvement of the RasRas-activated Rab5 guanine nucleotide exchange factor RIN2-Rab5 pathway in the hepatocyte growth factor-induced endocytosis of E-cadherin. J Biol Chem 2006; 281:10598-10609.

15 Syx D, Malfait F, Van Laer L, et al. The RIN2 syndrome: a new autosomal recessive connective tissue disorder caused by deficiency of Ras and Rab interactor 2 (RIN2). Hum Genet 2010; 128:79-88. 